\title{
Heart Sound Classification Based on Fractal Dimension and MFCC Features Using Hidden Markov Model
}

\author{
Mahbubeh Bahreini \\ Islamic Azad University \\ Ramin Barati ( $\sim$ barati.ramin@aut.ac.ir) \\ Islamic Azad University \\ Abbas Kamaly \\ Islamic Azad University
}

\section{Research Article}

Keywords: Heart sound, Fractal dimension, MFCC, Hidden Markov model

Posted Date: January 14th, 2022

DOI: https://doi.org/10.21203/rs.3.rs-1207404/v1

License: (c) (i) This work is licensed under a Creative Commons Attribution 4.0 International License.

Read Full License 


\title{
Heart Sound Classification based on Fractal dimension and MFCC Features using Hidden Markov Model
}

\author{
Mahbubeh Bahreini, Ramin Barati*, Abbas Kamaly \\ Department of Electrical Engineering, Shiraz Branch, Islamic Azad University, Shiraz, Iran
}

\begin{abstract}
Early diagnosis is crucial in the treatment of heart diseases. Researchers have applied a variety of techniques for cardiovascular disease diagnosis, including the detection of heart sounds. It is an efficient and affordable diagnosis technique. Body organs, including the heart, generate several sounds. These sounds are different in different individuals. A number of methodologies have been recently proposed to detect and diagnose normal/abnormal sounds generated by the heart. The present study proposes a technique on the basis of the Mel-frequency cepstral coefficients, fractal dimension, and hidden Markov model. It uses the fractal dimension to identify sounds S1 and S2. Then, the Mel-frequency cepstral coefficients and the first- and second-order difference Mel-frequency cepstral coefficients are employed to extract the features of the signals. The adaptive Hemming window length is a major advantage of the methodology. The S1-S2 interval determines the adaptive length. Heart sounds are divided into normal and abnormal through the improved hidden Markov model and Baum-Welch and Viterbi algorithms. The proposed framework is evaluated using a number of datasets under various scenarios.
\end{abstract}

Keywords- Heart sound, Fractal dimension, MFCC, Hidden Markov model

\section{Introduction}

Many individuals experience cardiovascular diseases across the world. It is possible to diminish the treatment cost and risk of such diseases through early diagnosis. Despite a lack of associations between the heart and abnormalities (e.g., chest pain), patients may become concerned about such abnormalities the diseases of the heart cannot be easily distinguished from other diseases; that is, heart abnormalities are not easily distinguished from common heart sounds [1]. It is important to diagnose heart conditions early and accurately. As a result, machine learning techniques have been recently exploited for heart disease diagnosis. Researchers collect cardiac data in a variety of manners, such as electrocardiogram (EEC) and photoplethysmogram (PPG) signals. These methods have strengths and weaknesses. A phonocardiogram (PCG) can also be employed to collect cardiac data. It utilizes sensitive microphones to record the sounds of the heart.

PCG is based on the assumption that heart sounds contain significant information that can be extracted to identify the heart health status. The distinction of normal and abnormal heart sounds diminishes stress in the patients and raises the likelihood of efficient and effective disease treatment. Efficient smartphone applications can be introduced and utilized for the detection of both normal and abnormal sounds of the heart. Hence, high-accuracy and simple techniques should be devised for the extraction of heart sound data [2].

The heart of a human may generate various sounds with different amplitudes and frequencies. Furthermore, age can influence the heart sound frequency and amplitude. Noise in the recorded heart sounds should also be taken into account. Vessel blood flow, breathing, and lungs induce such noise. It is necessary to remove noise prior to processing the recorded 
sounds. Moreover, the heart of a healthy individual generates different sounds from that of an individual with diseases [3]. A heartbeat generally involves two sounds, i.e., S1 and S2. There is a clear distinction between S1 and S2 in normal sounds of the heart. However, they cannot be easily distinguished in abnormal sounds. The high-frequency components of S2 are typically more than those of S1 [4].

A number of studies investigated the processing of heart sounds. They extracted various features from the sounds. Such studies employed either time-domain or frequency-domain features from the signals of the hearts. Furthermore, a number of works utilized both timeand frequency-domain features instead of statistical features. Accuracy is improved when a larger number of features are extracted; however, the learning algorithm would become more complex in such a case. As a result, fewer features or feature reduction should be utilized. Earlier works employed the Mel-frequency cepstral coefficients (MFCCs) in order to implement a heart sound classification into normal and abnormal [6]. Features were extracted using intrinsic mode functions (IMFs). As a result, fundamental cardiac sounds can be represented in numerous oscillating components to enhance algorithm accuracy [7]. Heart sound features were extracted using Shannon entropy and IMFs [8]. To classify the sounds of the heart, previous studies employed a variety of statistical features [9]. To diagnose heart sound abnormalities, various time-domain features were exploited [10].

Numerous works employed artificial neural networks (ANNs), convolutional neural networks (CNNs), and support vector machines (SVMs) in the literature [11, 12, 13]. However, recent studies have been focused on hidden Markov models (HMMs). This approach assumes the system under study as a Markov process involving hidden states. HMMs could be described to be the simplest variation of dynamic Bayesian networks. HMM advantages include [14]:

- Robust statistical foundations,

- High learning efficiency (direct learning from raw sequence data is possible),

- High flexibility in sequence profile generalization, and

- A locally learnable form.

HMM techniques have been utilized in many studies for heart sound detection. An HMM was employed to classify heart sounds [15]. Heart sounds were segmented and classified using a Markov-switching model. They segmented heart sound cycles and extracted features from the segments [16]. To segment heart sounds, previous studies exploited a semi-Markov model. Likewise, the heart sound cycles were segmented into S1, systolic period (Sys), S2, and diastolic period (Dias) [17]. Lung and heart sounds were segmented using hidden Markov-Gaussian mixture models. To detect normal and abnormal sounds, MFCC features and quartiles were employed [18].

Previous studies mostly segmented heart sound cycles into four groups and could not develop effective and efficient approaches to detect heart sounds. Furthermore, signals were processed using fixed-length windows in earlier works. The present study utilizes the fractal dimension to divide heart sounds into four segments, independently extracting the features of each segment through MFFCs. The major novelty of the proposed methodology lies in the extraction of various features for each segment. This paper proposes an adaptive Hamming window, and an HMM is utilized to calculate the window length. The HMM is implemented to distinguish normal and abnormal heart sounds. To utilize the proposed method in the 
form of a smartphone application, the present study employed the standard HMM. Moreover, improved Viterbi and Baum-Welch algorithms were adopted for sound detection to enhance the efficiency and effectiveness of the proposed method. This improves outlier and noise robustness. The remainder of the study is organized as follows: Section 2 describes the proposed method; Section 3 provides and discusses the results; and, Section 4 concludes the work.

\section{Proposed Model}

The recorded sounds of the heart contain noise. The noise was removed from the sounds using the 5-order band-pass Butterworth filter at 25 to $400 \mathrm{~Hz}$. The fractal dimension was utilized to process the denoised signals so that sounds S1 and S2 could be detected before calculating the length of the Hamming window through the S1-S2 time interval. The MFCC features and first and second MFCC feature differences of the windows were extracted. Eventually, normal and abnormal heart sounds were detected through the HMM and improved Viterbi and Baum-Welch algorithms. The windows were set to be long enough to involve all four segments (i.e., S1, Sys, S2, and Dias). The proposed method is depicted in the form of a flowchart in Fig. 1.

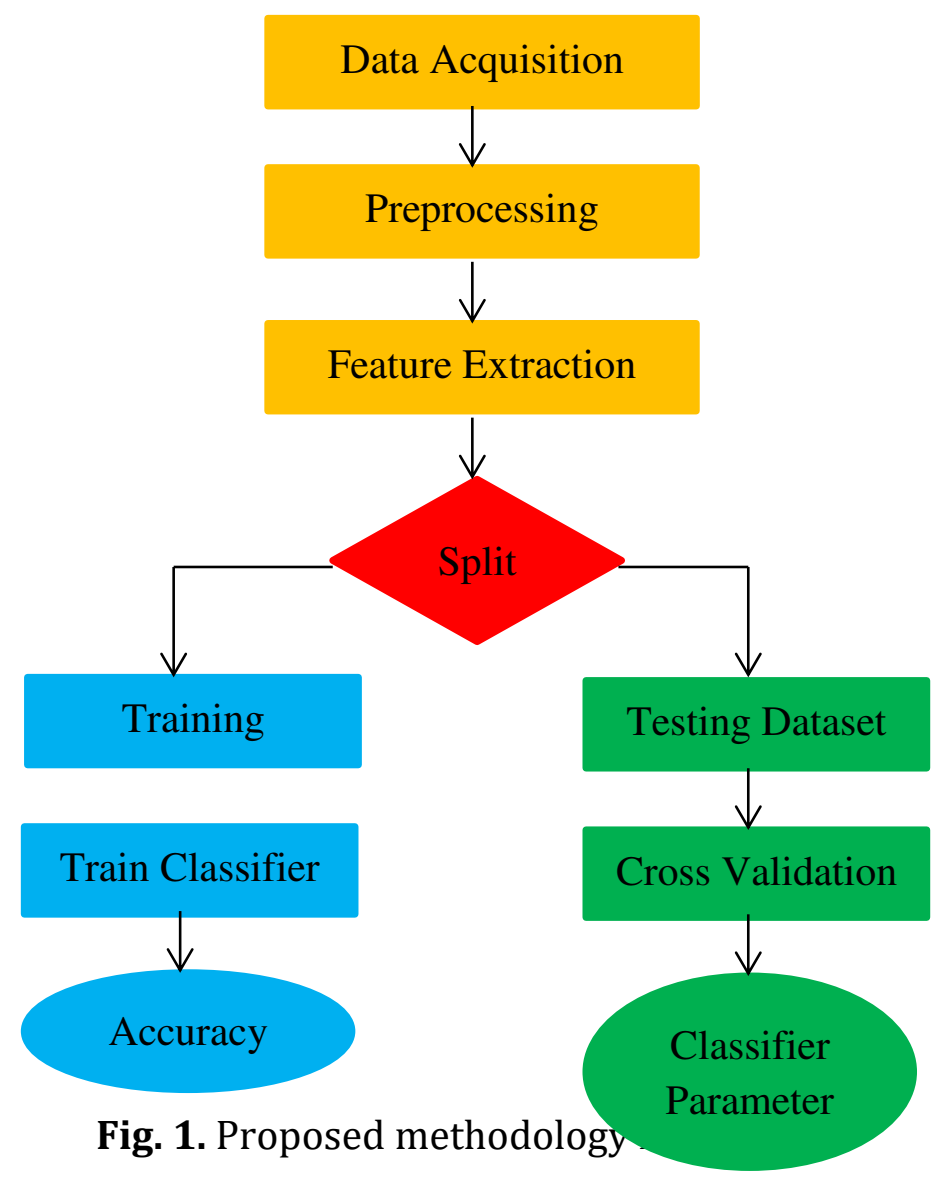

\section{1- Extract Heart sounds}

The use of the fractal dimension and a wavelet transform for the detection of S1 and S2 is described here. The length of the Hamming window should be calculated to extract signal features. Wavelet transform is written as [19]: 


$$
\psi_{\alpha, \beta}(t)=\frac{1}{\sqrt{\alpha}} \psi\left(\frac{t-\beta}{\alpha}\right)
$$

in which $\psi(\mathrm{t})$ denotes the mother wavelet, while $\alpha$ and $\beta$ stand for the dilation scale and translation parameter, respectively. Earlier works have developed a variety of mother wavelets. The present work adopted a Ricker mother wavelet. The wavelet transform decomposes a signal into a few scales using a filter bank. At each transform level, the low components are separated from the high components for signal resolution improvement and noise removal. The input signal and levels 3 and 5 are illustrated for the normal and abnormal heart sounds in Fig. 1. This paper utilizes level 5 as the fractal dimension input [20]. Fractal sets represent many geometrically complex natural phenomena, where the fractal dimension defines the fracture character. To implement the fraction dimension approach, a sliding window is employed. The length of the sliding window is given by:

(2) $W=\operatorname{int}\left(0.05 F_{s}\right)$

in which int (.) represents the integer component of argument, while $F_{s}$ stands for the frequency of sampling. It should be noted that 0.05 denotes an adjustable parameter. This work adopted the Petrosian fractal dimension for fractal dimension estimation [21]. For a signal consisting of points $\left\{\mathrm{y}_{1}, \mathrm{y}_{2}, \ldots, \mathrm{y}_{\mathrm{N}}\right\}$ (where $\mathrm{N}$ denotes the sample population), the fractal dimension is given by:

$$
F D=\frac{\log _{10} N}{\log _{10} N+\log _{10}\left(\frac{N}{N+0.4 N \mathrm{~V}}\right)}
$$

in which $\mathrm{N}_{\Delta}$ represents the total number of changes in adjacent symbol $\xi_{\mathrm{i}}$ :

(4) $N \mathrm{~V}=\sum_{i=1}^{N-2}\left|\frac{\zeta_{i+1}-\zeta_{i}}{2}\right|$

where

(5) $\zeta_{i}=\left\{\begin{array}{ll}+1, & y_{i}>\text { mean }(y) \\ -1, & y_{i} \leq \text { mean }(y)\end{array}, \quad i=1,2, \ldots, N\right.$

This study implemented the fractal dimension on wavelet signals. The iteration begins with a threshold operation given by [20]:

$$
S f d_{j}^{i}=\left\{\begin{array}{lc}
F D_{j}^{i}, & F D_{j}^{i}>\mu^{i}+\sigma^{i} \\
1, & \text { o.w }
\end{array}, i=1,2, \ldots, L ; j=1,2, \ldots, M\right.
$$

in which $\mu$ is the mean, while $\sigma$ is the standard deviation of the fractal dimension vector. Furthermore, $\mathrm{M}$ and $\mathrm{L}$ stand for the numbers of fractal coefficients and iterations, respectively. Through Eqs. (3) and (6), one can define a new variable as:

(7) $z^{i}=F D_{j}^{i}-S_{j}^{i}+\mu^{i}$

Iterations continue until the discontinuance criterion has been met:

$$
F=\left|E\left\{\left(z^{i}\right)^{2}\right\}-E\left\{\left(z^{i-1}\right)^{2}\right\}\right|
$$

in which $\mathrm{E}($.$) denotes the expectation. Thus,$

$$
D_{j}^{i}=\sum_{k=1}^{L} S f d^{k}-(L-1), i=1,2, \ldots, L ; j=1,2, \ldots, M
$$


Eventually, the output signal is given by:

$$
S_{B T H}^{i}= \begin{cases}1, & D_{j}^{i} \neq 1 \\ 0, & D_{j}^{i}=1\end{cases}
$$

Fig. 2 illustrates the fractal algorithm in detail. As can be seen, the fractal method is capable of converting the sound signals of the heart into binary signals. The output signal is 1 for sounds S1 and S2 and zero for the remaining components. The signals of S1 and S2 are identified using the SBTH signal. Considering that systolic duration is shorter than diastolic duration in cardiac cycles, $\mathrm{S} 1$ and $\mathrm{S} 2$ are detected as:

$$
S(2 i+1)-S(2 i)<S(2 i+3)-S(2 i+2)
$$

Once sounds S1 and S2 have been predicted, it is possible to divide the heart signal into four segments. Fig. 3 illustrates heart sound segmentation. It should be mentioned that segment lengths could vary.

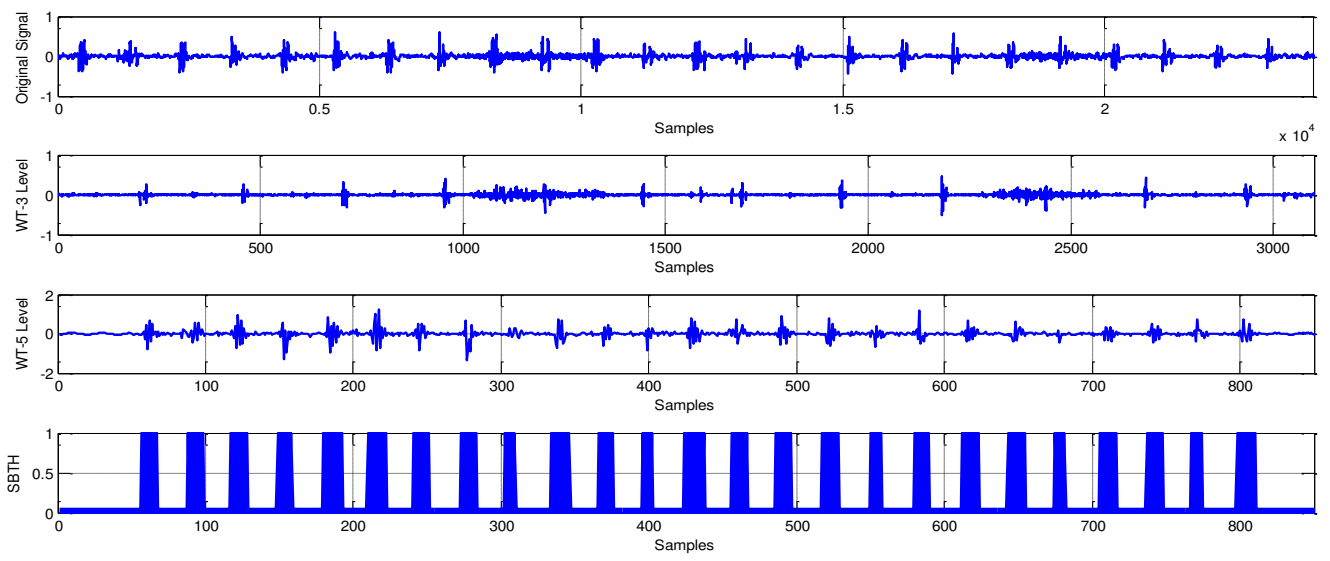

(a)
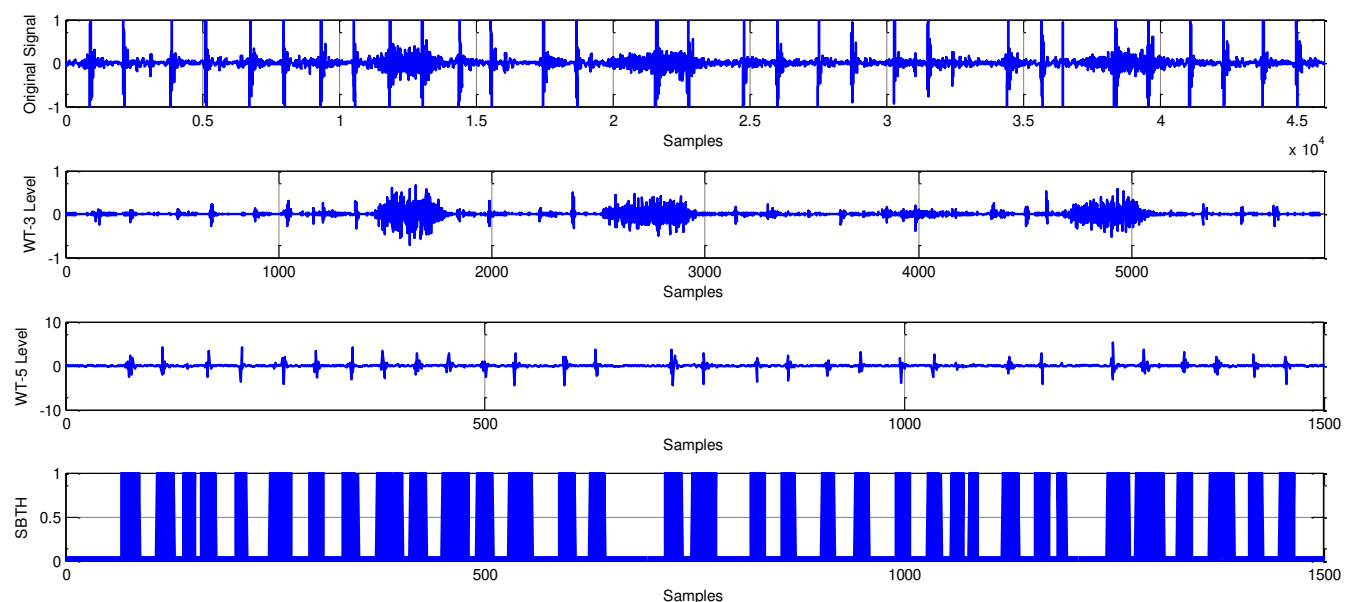

(b)

Fig. 2. Wavelet decomposition and SBTH for (a) normal and (b) abnormal sounds 


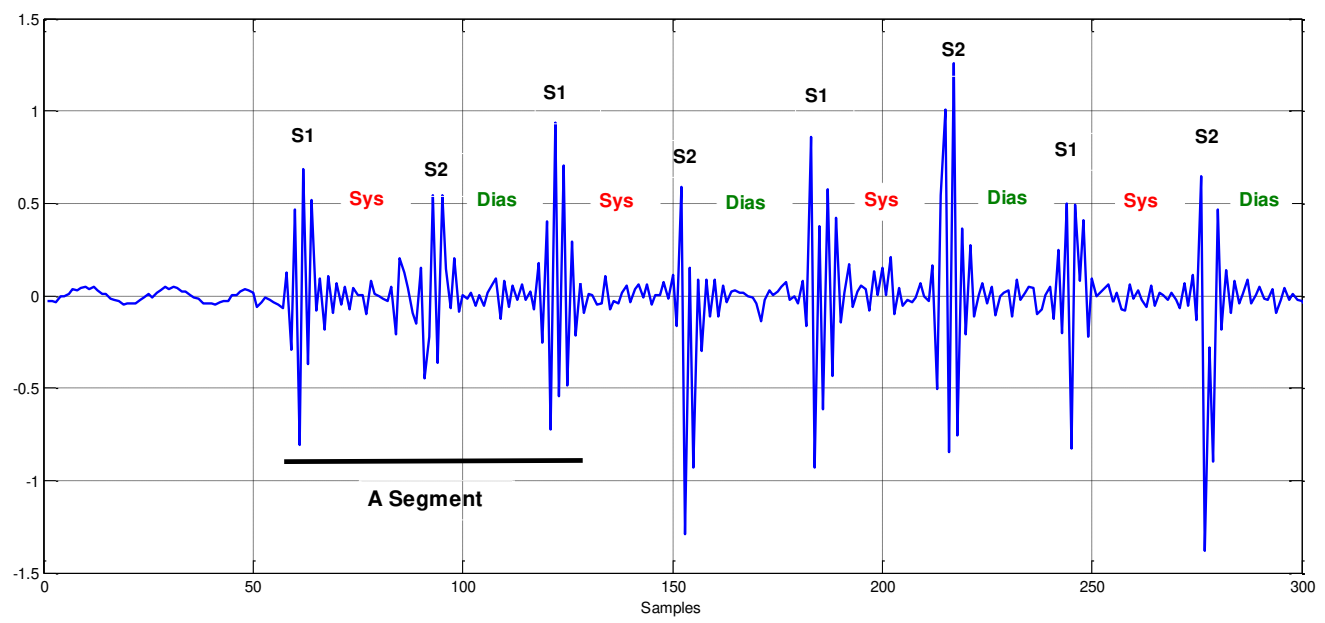

Fig. 3. Heart sound segmentation

\section{2- Extraction of MFCC features}

The short-term sound power spectrum is represented on the basis of a linear cosine transform of a log power spectrum at a nonlinear frequency Mel scale by the Mel-frequency cepstrum (MFC). MFC is employed to represent nonlinear associations between the frequency of the heart sound and the human ear. It is written as [22]:

$$
\operatorname{Mel}(f)=2595 \log _{10}\left(1+\frac{f}{700}\right)
$$

in which $f$ denotes the actual sound frequency. MFCCs constitute an MFC. They are obtained from a cepstral sound signal representation. Frequency bands have equal spacing at the Mel scale in the MFC. The human auditory response closer to the linear frequency bands is employed in normal cepstrum. The Hamming window is used to frame the sound so that MFCCs could be calculated. The present work proposes an adaptive length of the Hamming window. The fractal dimension is utilized to obtain the window length. For $\mathrm{N}$ samples, the discrete Fourier transform is employed to calculate the signal spectrum as:

$$
X(k)=\sum_{n=0}^{N-1} x(n) \exp \left(-\frac{2 \pi n k}{N}\right), \quad 0 \leq n, \quad k \leq N-1
$$

The signal power spectrum is found as:

$$
P(k)=\frac{1}{N}|X(k)|^{2}
$$

The Mel spectrum is derived by applying filter banks to the power spectrum. The band filter may be formulated as:

$$
H(k)= \begin{cases}0, & k<f(m-1) \\ \frac{k-f(m-1)}{f(m)-f(m-1)}, & f(m-1) \leq k \leq f(m) \\ \frac{f(m+1)-k}{f(m+1)-f(m)}, & f(m) \leq k \leq f(m+1) \\ 0, & k>f(m+1)\end{cases}
$$

in which $\mathrm{f}(\mathrm{m})$ denotes the Mel triangle filter center frequency. MFCCs could be found as: 
(16) $\operatorname{MFCC}(n)=\sum_{m=0}^{N-1} E(m) \cos \left(\frac{\pi n(m-0.5)}{M_{f}}\right), \quad n=1,2, \ldots, L_{m}$

in which $\mathrm{Lm}_{\mathrm{m}}$ stands for the MFFC order, while $\mathrm{M}_{\mathrm{f}}$ stands for the filter bank count. Also, the logarithmic energy spectrum is formulated as:

$$
E(m)=\ln \left[\sum_{k=0}^{N-1} P(k) H(k)\right], \quad 0 \leq m \leq M_{f}
$$

The static features of MFCCs could be calculated using Eq. (16). The dynamic features of MFCCs are represented by the first- and second-order difference MFCC. These dynamic features provide beneficial knowledge. $\triangle \mathrm{MFCC}$ represents the first-order difference MFCC:

$$
\triangle M F C C=\frac{1}{\sqrt{\sum_{i=-k}^{k} i^{2}}} \sum_{i=-k}^{k}(i \times M F C C(n+i))
$$

Moreover, $\Delta^{2}$ MFCC demonstrates the second-order difference MFCC:

$$
\Delta^{2} M F C C=\frac{1}{\sqrt{2 \sum_{i=-k}^{k} i^{2}}} \sum_{i=-k}^{k}(i \times \Delta M F C C(n+i))
$$

The MFCC, $\triangle$ MFCC, and $\triangle^{2}$ MFCC features are illustrated for the normal and abnormal sounds of the heart in Fig. 4. As can be seen, a total of fourteen features were extracted from MFCC, $\triangle$ MFCC, and $\triangle^{2}$ MFCC. 

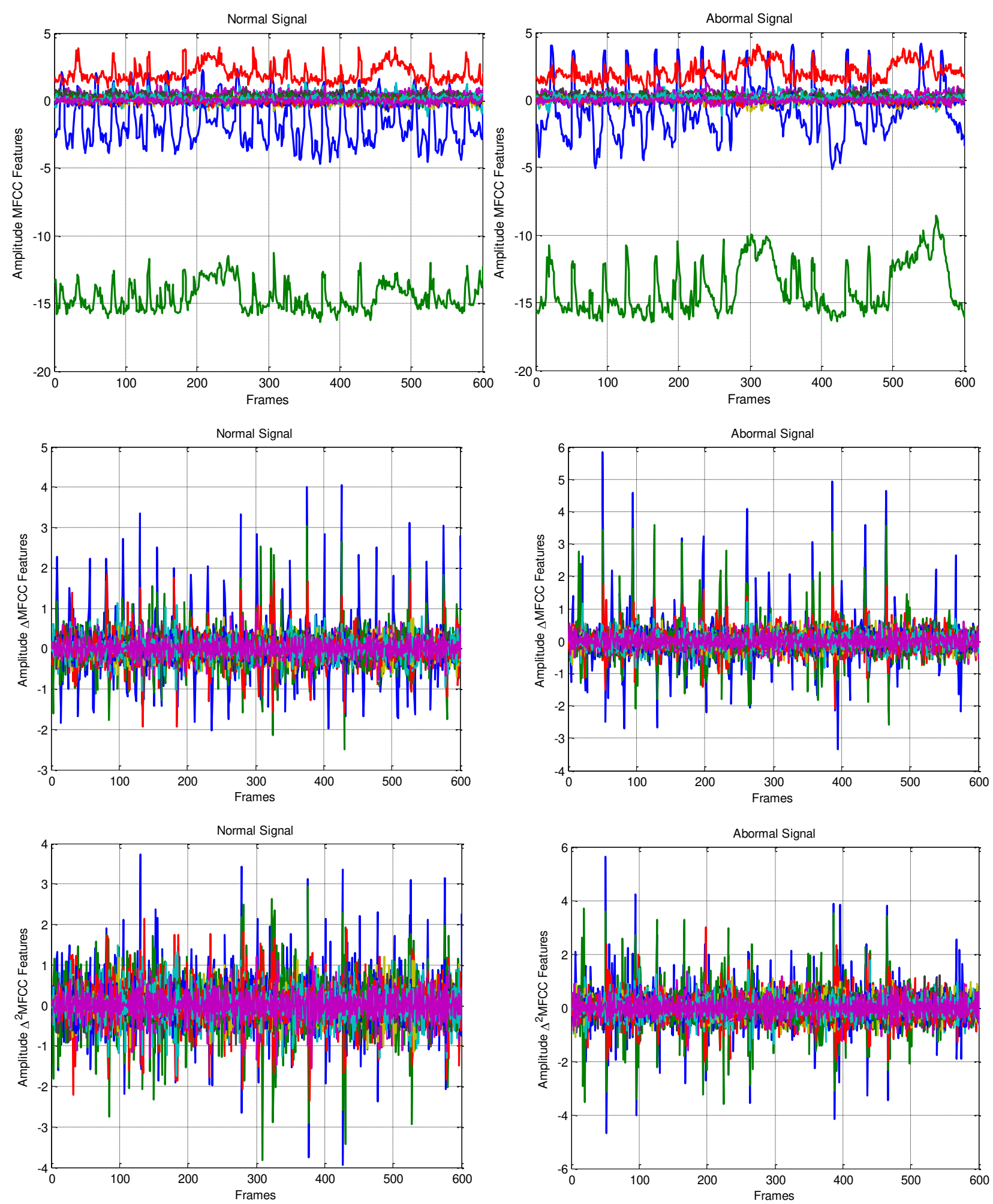

Fig. 4. MFCC, $\triangle \mathrm{MFCC}$, and $\Delta^{2} \mathrm{MFCC}$-extracted features of normal and abnormal heart

\section{3- Hidden Markov model} sounds 
Markov processes of high complexity may be simulated by hidden Markov models. This simulation leads to states on the basis of the possible observation distribution. The probability of a given observation set member for a certain possible latent state which could follow a probability distribution is determined by the output probability. The observation variable nature determines the number of observation set members [23].

An HMM involves a finite set of hidden possible states $S=\left\{\mathrm{s}_{1}, \mathrm{~s}_{2}, \ldots, \mathrm{S}_{\mathrm{N}}\right)$. A state stands for a class of heart sounds. The present study included two states: normal and abnormal.

The state transition probability is a measure of the probability of a transition from state $i$ to $j$. It is written as:

$$
a_{i, j}=\operatorname{Pr}\left(s_{j} \mid s_{i}\right), \quad i=1,2 ; j=1,2 ; a_{i j} \geq 0 ; \sum_{j=1}^{2} a_{i j}=1
$$

The matrix form of the transition probability is shown as:

$$
A=\left[\begin{array}{ccc} 
& 1 & 2 \\
1 & a_{11} & a_{12} \\
2 & a_{21} & a_{22}
\end{array}\right]
$$

in which 1 and 2 stand for normal and abnormal sounds of the heart, respectively.

$\mathrm{O}=\left\{\mathrm{x}_{1}, \mathrm{x}_{2}, \ldots, \mathrm{X}_{\mathrm{F}}\right\}$ denotes the number of observations, in which $\mathrm{F}$ is the observation (feature) count. A total of forty-two features are extracted from the sounds at each signal and each frame (fourteen features from MFCCs and first- and second-order difference MFCCs each). The features of normal sounds differ from those of abnormal sounds.

For a model at state $S_{\mathrm{j}}$, the emission function of state $i$ stands for the emission probability of an observation. It is given by:

$$
b_{j k}=\operatorname{Pr}\left(O=x_{k} \mid S=s_{j}\right), 1 \leq k \leq F ; j=1,2 ; b_{j k} \geq 0 ; \sum_{k=1}^{F} b_{j k}=1
$$

The matrix form of the emission function is shown as:

$$
B=\left[\begin{array}{ccccc} 
& 1 & 2 & \mathrm{~K} & F \\
1 & b_{11} & b_{12} & \mathrm{~K} & b_{1 F} \\
2 & b_{21} & b_{22} & \mathrm{~K} & b_{F F}
\end{array}\right]
$$

It should be noted that $\pi$ denotes the initial probability of a state. Also, $\pi_{i}$ denotes the probability that state $i$ is the initial state. Eventually, one can simulate the HMM using triplets $\lambda=(\mathrm{A}, \mathrm{B}, \pi)$. 
Data: sequence: O, partially label X

Result: best A, best B

Initialization: $\lambda=(\mathrm{A}, \mathrm{B}, \pi)$

Best Accuracy $\leftarrow 0$

While (Not coverage nor iteration < Iteration_max)

Calculate $\alpha$ and $\beta$ by following equations

$\alpha_{i}(1)=\pi b_{i}\left(O_{1}\right)$

$\alpha_{i}(t+1)= \begin{cases}b_{i}\left(O_{t+1}\right) \sum_{j=1}^{N} \alpha_{j}(t) a_{i j}, & L\left(O_{t+1}\right)=0 \text { or } L\left(O_{t+1}\right)=i \\ 0, & L\left(O_{t+1}\right) \neq 0 \text { or } L\left(O_{t+1}\right) \neq i\end{cases}$

$\beta_{i}(T)=1$

$\beta_{i}(t)=\left\{\begin{array}{l}\sum_{j=1}^{N} \beta_{j}(t+1) a_{i j} b_{j}(O(t+1)), \quad L\left(O_{t+1}\right)=0 \text { or } L\left(O_{t+1}\right)=i \\ 0, \quad L\left(O_{t+1}\right) \neq 0 \text { or } L\left(O_{t+1}\right) \neq i\end{array}\right.$

Calculate $\gamma$ and $\xi$ by using the following equations

$$
\begin{aligned}
& \gamma_{i}(t)=\operatorname{Pr}(X(t)=i \mid \theta, O)=\frac{\operatorname{Pr}(X(t)=i \mid \theta)}{\operatorname{Pr}(O \mid \theta)}=\frac{\alpha_{i}(t) \beta_{i}(t)}{\sum_{j=1}^{N} \alpha_{j}(t) \beta_{j}(t)} \\
& \xi_{i j}(t)=\frac{\operatorname{Pr}(X(t)=i, X(t+1)=j, O \mid \theta)}{\operatorname{Pr}(O \mid \theta)}=\frac{\alpha_{i}(t) a_{i j} \beta_{j}(t+1) b_{j}(O(t+1))}{\operatorname{Pr}(O \mid \theta)}
\end{aligned}
$$

Update $\pi^{*}, \mathrm{~A}^{*}$ and $\mathrm{B}^{*}$ as follows:

$$
\begin{aligned}
& \pi(i)^{*}=\gamma_{i}(1) \\
& a_{i j}^{*}=\frac{\sum_{t=1}^{T-1} \xi_{i j}(t)}{\sum_{t=1}^{T-1} \gamma_{i}(t)} \\
& b_{i}^{*}\left(o_{k}\right)=\frac{\sum_{t=1}^{T-1} \gamma_{i}(t) I_{O(t)=o_{k}}}{\sum_{t=1}^{T-1} \gamma_{i}(t)}
\end{aligned}
$$

$\mathrm{X}^{*} \leftarrow \operatorname{Decoding}\left(\pi^{*}, \mathrm{~A}^{*}, \mathrm{~B}^{*}, \mathrm{O}\right)$

newAccuracy $\leftarrow$ Accuracy $\left(X^{*}, X\right)$

if (newAccuracy > BestAccuracy)

$$
\begin{aligned}
& \pi \leftarrow \pi^{*} \\
& \text { best } A \leftarrow A^{*} \\
& \text { bestB } \leftarrow B^{*}
\end{aligned}
$$

Fig. 5. Improved Baum-Welch algorithm pseudocode

It is required to train the HMM for the calculation of $A, B$, and $\pi$. The training phase was implemented using the improved Baum-Welch algorithm (as it is simple) [24] and heart 
sound data. Then, the HMM is evaluated using a forward-backward algorithm. The improved Baum-welch algorithm involves the partial labeling of training sequences, where possible hidden state paths are constrained in expected value calculation. This procedure affects the detection of the signals of features in biological datasets, as depicted in Fig. 5.

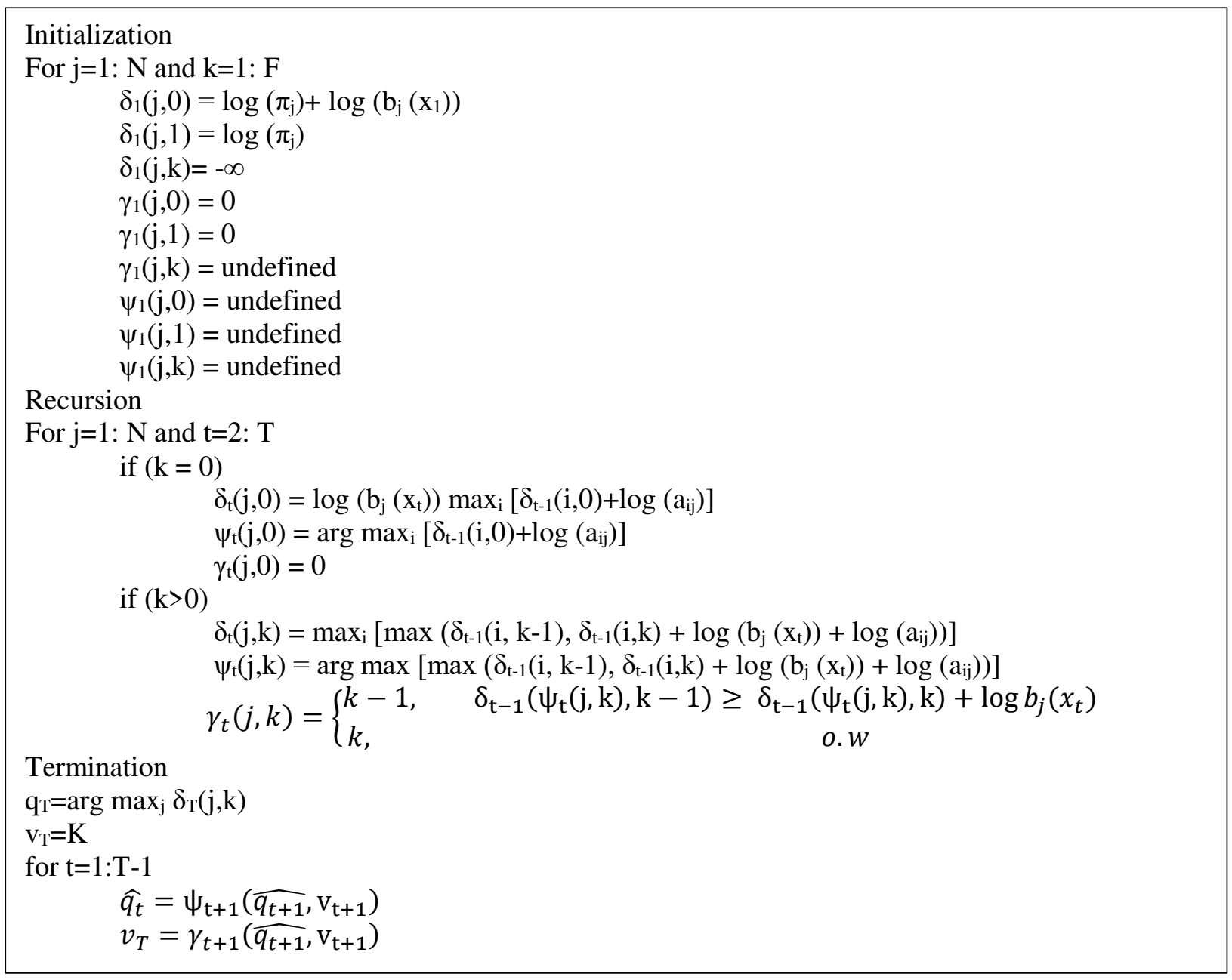

Fig. 6. Improved Viterbi algorithm pseudocode

The likelihood scores of HMMs were calculated on the basis of the state sequence of the highest probability using the Viterbi algorithm, considering an unknown test heart sound beat. However, the outlier robustness of the Viterbi algorithm might decline. Thus, the present study adopted the improved Viterbi algorithm for likelihood score calculation, as shown in Fig. 6 [25]. The limited number of corruptions does not affect the best state sequence through a focus on finding the best path by eliminating $k$ worst observations in the improved Viterbi algorithm. It should be noted that $\delta_{\mathrm{t}}(\mathrm{j}, \mathrm{k})$ denotes the log robust likelihood of the best path at time up to $t-1$, and $\mathrm{q}_{\mathrm{j}}=\mathrm{t}$ after the elimination of $\mathrm{F}$ observations.

\subsection{Evaluation metrics}

The present work adopted accuracy, precision, and sensitivity as the evaluation metrics. Accuracy represents to what extent the calculations are close to the real value, precision 
represents to what extent the calculations are similar to each other, and sensitivity refers to a fraction of the total actually retrieved relevant instances.

$$
\begin{aligned}
\text { Accuracy } & =\frac{T P+T N}{T P+T N+F P+F N} \\
\text { Precision } & =\frac{T P}{T P+F P} \\
\text { Sensitivity } & =\frac{T P}{T P+F N}
\end{aligned}
$$

in which TP stands for the true positive, FP denotes the false positive, TN represents the true negative, and FN is the false negative.

\section{Results}

The proposed framework was implemented on four datasets to evaluate its performance. Dataset 1 was the 2016 PhysioNet/CinC dataset [26], Dataset 2 was the Peterjbentley dataset [27], Dataset 3 was the Shiraz University Fetal Heart Sounds dataset [28], and Dataset 4 was the Kaggle/heartbeat sound dataset [29]. Moreover, a number of scenarios were implemented to evaluate the influences of MFCCs and first- and second-order difference MFCCs on performance. The performances of a number of HMMs were also evaluated. 70\% of each dataset was randomly selected to train the model, while the remaining $30 \%$ was utilized to test the model. The average output of ten distinct executions was reported as the final result.

Fig. 7 illustrates the performance evaluation results. Mode 1 extracted sound features using no classification based on the fractal dimension. The other models, however, employed the fractal dimension to detect S1 and S2. Model 2 extracted MFCC features. Model 3 extracted not only MFCC but also $\triangle$ MFCC features. Model 4 extracted $\Delta^{2}$ MFCC features instead of MFCC and $\triangle$ MFCC. As can be seen, model 4 had the highest evaluation metrics (i.e., accuracy, precision, and sensitivity). Model 4 outperformed models 1 and 2. Furthermore, model 1 was found to have the lowest performance based on the evaluation metrics. Therefore, it can be said that model efficiency and effectiveness improve by using adaptive frame segmentation and extracting a larger number of features.
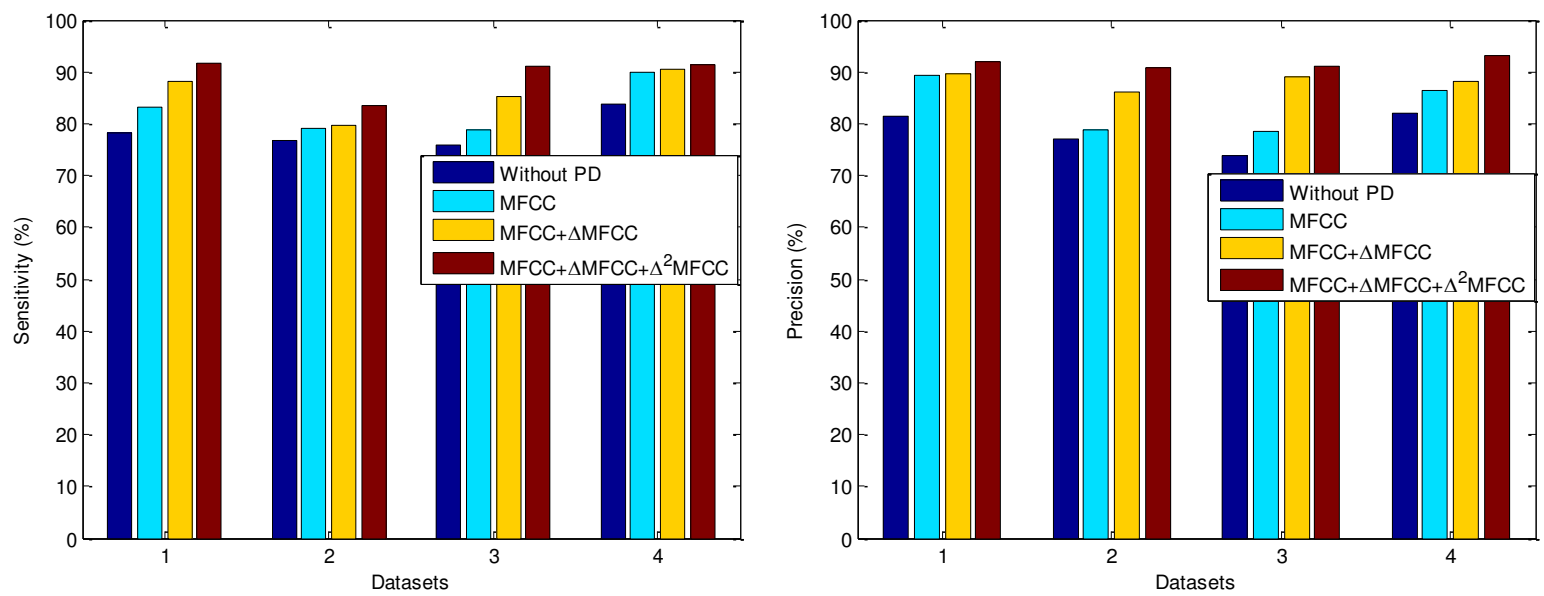


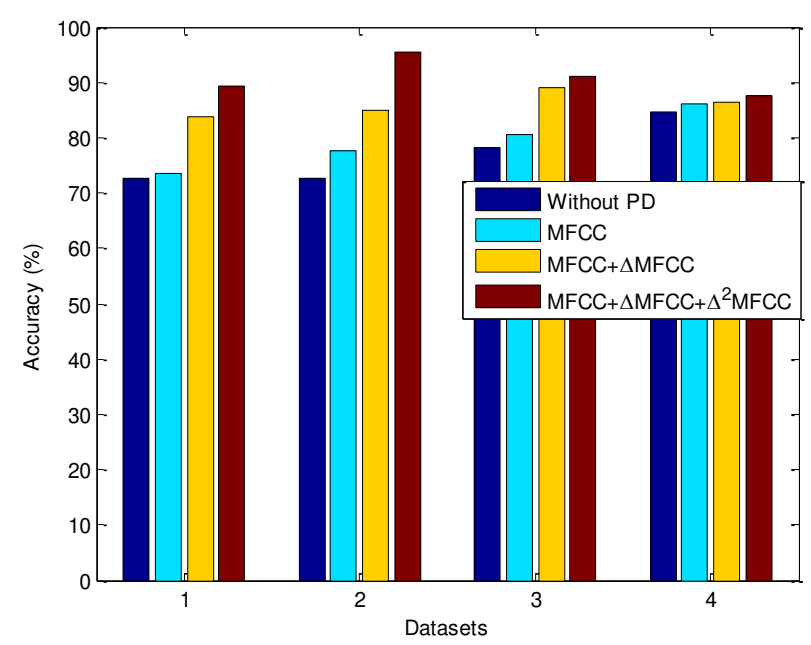

Fig. 7. Performance evaluation results

Fig. 8 compares HMMs in performance. In the performance evaluation of the models, a low signal SNR is assumed (0 dB). It should be noted that the Gaussian mixture model-HMM is denoted by GMM-HMM, while the hidden-semi Markov model is represented by HSMM. As can be seen, the models had a performance order of improved HMM $>$ HSMM $>$ GMM$\mathrm{HMM}>\mathrm{HMM}$.
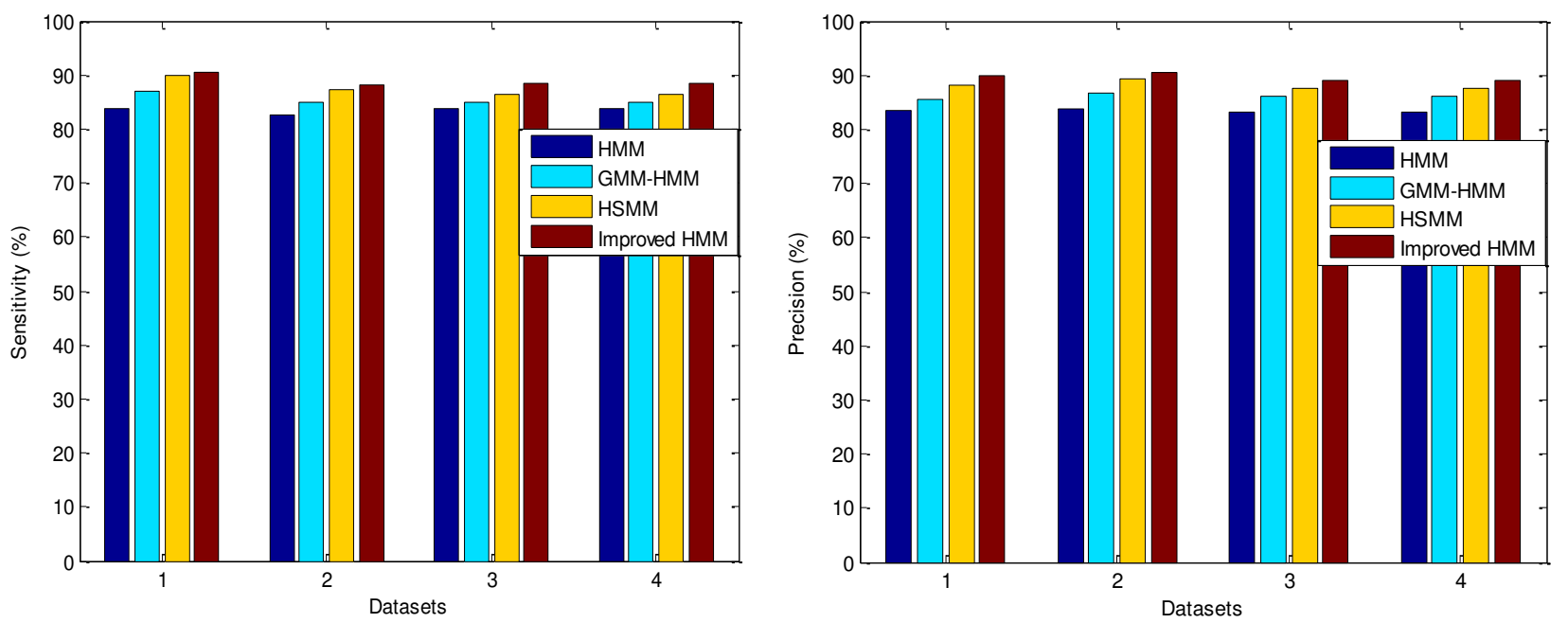


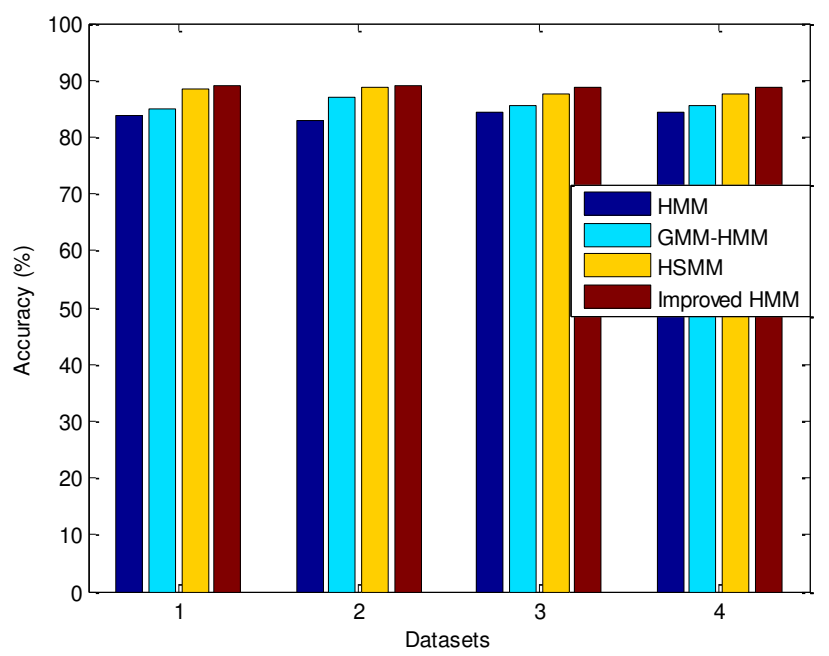

Fig. 8. Performance comparison of HMMs

Fig. 9 compares the evaluation metrics of the proposed model to previous studies. As can be seen, the proposed model was found to outperform the methodologies proposed in earlier works.
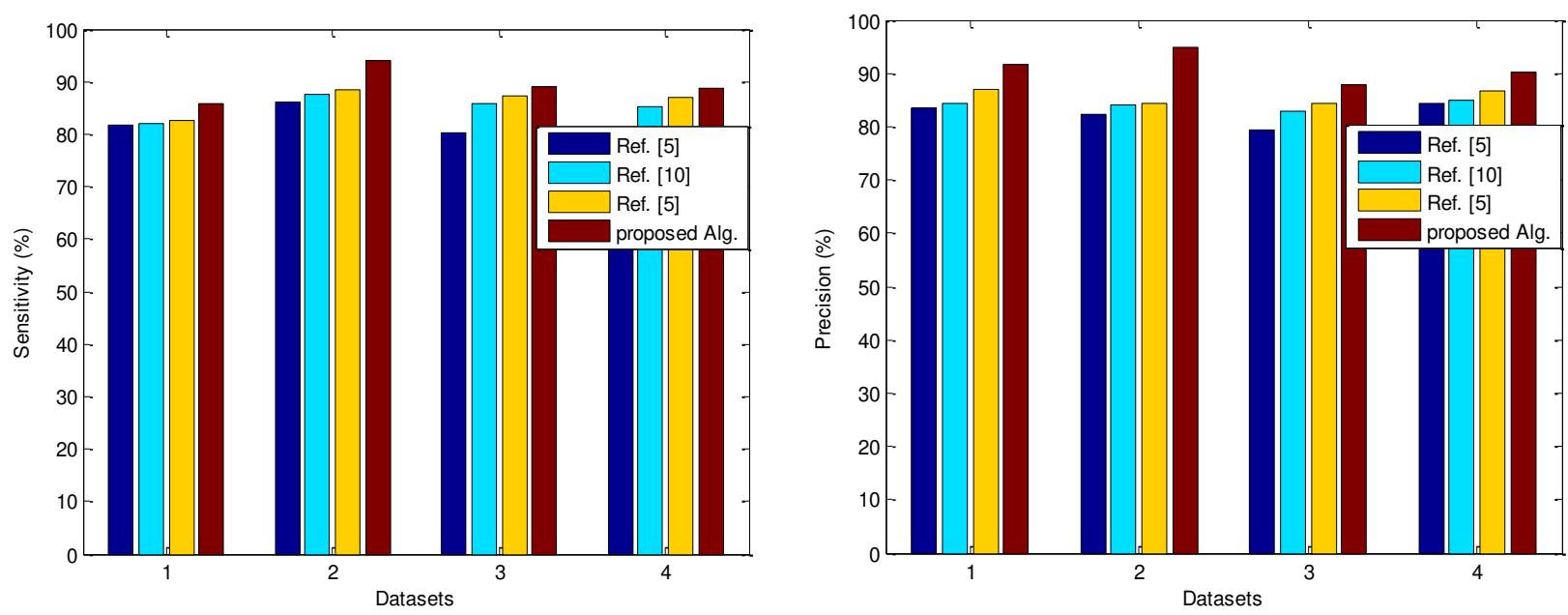


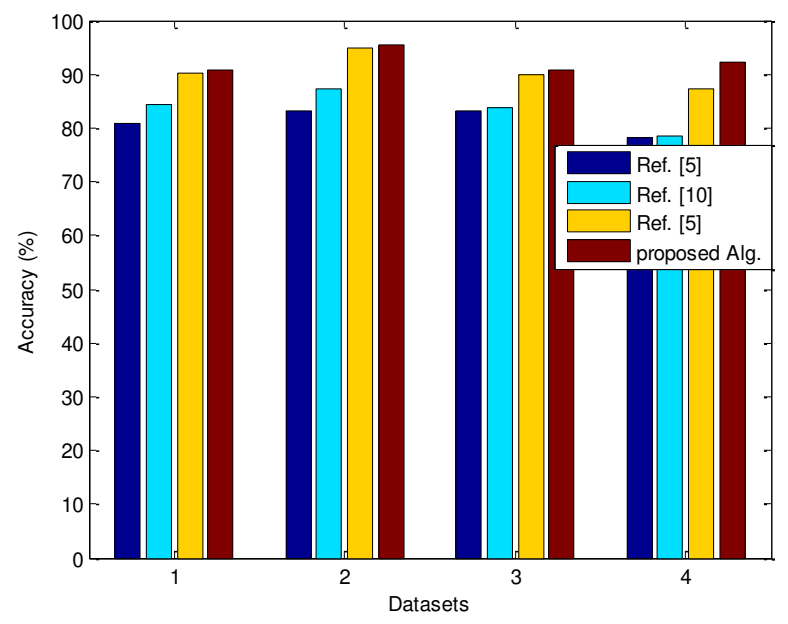

Fig. 9. Performance comparison of various algorithms

\section{Conclusion}

The present study proposed a new methodology on the basis of improved MFCC and HMM features. Sounds S1 and S2 were extracted using the fractal dimension, calculating the length of the Hamming window through the S1-S2 time interval. The S1-S2 time interval differed for different heart cycles and different individuals. Therefore, the proposed methodology was based on an adaptive length of the Hamming window. Static and dynamic sound features were extracted using MFFCs, first-order difference MFFCs, and second-order MFFCs. The features were introduced to the HMM as inputs. The improved Viterbi and Baum-Welch algorithms enhanced the efficiency of the HMM. The model was implemented on four different datasets to evaluate its performance. It was found that the proposed methodology outperformed earlier works. Moreover, the improved HMM was found to be more efficient than the standard HMM, GMM-HMM, and HSMM. The extraction of static and dynamic features of MFCCs and the fractal dimension-based segmentation of signals were found to enhance the performance of the heart sound detection process in precision, accuracy, and sensitivity

\section{References}

[1] Zhang W., Han J, Deng S., Abnormal heart sound detection using temporal quasi-periodic features and long short-term memory without segmentation, Biomedical Signal Processing and Control 2019:53 53

[2] Tsao Y., et. Al., Robust S1 and S2 heart sound recognition based on spectral restoration and multi-style training, Biomedical Signal Processing and Control 2019:49

[3] Kamson A. P., Sharma L. N, Dandapat S., Multi-centroid diastolic duration distribution based HSMM for heart sound segmentation, Biomedical Signal Processing and Control 2019: 48

[4] Li J., Ke L., Du Q., Classification of Heart Sounds Based on the Wavelet Fractal and Twin Support Vector Machine, entropy, 2019:21

[5] Altuve M., Suárez L., Ardila J., Fundamental heart sounds analysis using improved complete ensemble EMD with adaptive noise, Biocybernetics and Biomedical Engineering 2020: 4

[6] Eslamizadeh G., Barati R., Heart murmur detection based on wavelet transformation and a synergy between artificial neural network and modified neighbor annealing methods, Artificial intelligence in medicine 2017: 78 
[7] Altuve M., Suárez L., Ardila J., Fundamental heart sounds analysis using improved complete ensemble EMD with adaptive noise, Biocybernetics and Biomedical Engineering 2020: 4

[8] Zhang W., Han J., Deng S., Heart sound classification based on scaled spectrogram and tensor decomposition, Expert Systems with Applications, 2017: 84

[9] Chen P., Zhang Q., Classification of heart sounds using discrete time-frequency energy feature based on S transform and the wavelet threshold denoising, Biomedical Signal Processing and Control 2020: 57

[10] Badlaoui O. E., Benba A., Hammouch A., Novel PCG Analysis Method for Discriminating Between Abnormal and Normal Heart Sounds IRBM 2019

[11] Saqlain Shah S. M., Ali Shah F., Hussain S. A., Batool S., Support Vector Machines-based Heart Disease Diagnosis using Feature Subset, Wrapping Selection and Extraction Methods, Computers and Electrical Engineering, 2020: 84.

[12] Cathers I., Neural network assisted cardiac auscultation, Artif. Intell. Med. 7 1995: 1

[13] Chen P., Zhang Q., Classification of heart sounds using discrete time-frequency energy feature based on $\mathrm{S}$ transform and the wavelet threshold denoising, Biomedical Signal Processing and Control 2020: 57

[14] Springer D., Tarassenko L., Support vector machine hidden semi- Markov model-based heart sound segmentation, Comput Cardiol, 2014.

[15] P. Sedighian, et al., "Pediatric heart sound segmentation using Hidden Markov Model," in Proc. 36th Annu. Int. Conf. IEEE Eng. Med. Biol. Soc., 2014

[16] Noman F., et. al., A Markov-Switching Model Approach to Heart Sound Segmentation and Classification, IEEE Journal of Biomedical and Health Informatics 2020: 24

[17] Maruotti A., Petrella L., Sposito L., Hidden semi-Markov-switching quantile regression for time series, Computational Statistics and Data Analysis 2021: 159

[18] Quartiles and Mel Frequency Cepstral Coefficients Vectors in Hidden Markov-Gaussian Mixture Models Classification of Merged Heart Sounds and Lung Sounds Signals, International Conference on High Performance Computing \& Simulation (HPCS), 2015

[19] Dautov. C. P., Özerdem. M. S., Wavelet transform and signal denoising using Wavelet method, 26th Signal Processing and Communications Applications Conference (SIU), 2018.

[20] Juniati D., Khotimah C., Wardani D. E. K., Budayasa K., Fractal dimension to classify the heart sound recordings with KNN and fuzzy c-mean clustering methods, The 2nd International Joint Conference on Science and Technology (IJCST) 2018

[21] Hamidi M., Ghassemian H., Imani M., Classification of heart sound signal using curve fitting and fractal dimension, Biomedical Signal Processing and Control 2018: 39

[22] Martinez J, Perez H, Escamilla E, Suzuki M. M. Speaker recognition using Melfre-quency cepstral coefficients (MFCC) and vector quantization (VQ) techniques. In: 22nd international conference on electrical communications and comput-ers; 2012

[23] Li J., Lee J. Y., Liao L., A new algorithm to train hidden Markov models for biological sequences with partial labels, BMC Bioinformatics, 2021: 22

[24] Scheffer T, Decomain C, Wrobel S. Active hidden Markov models for information extraction. In: International symposium on intelligent data analysis. Springer; 2001

[25] Siu M., Chan A., A Robust Viterbi Algorithm Against Impulsive Noise With Application to Speech Recognition, IEEE transactions on audio, speech, and language processing, 2006: 14

[26] https://physionet.org/content/challenge-2016/1.0.0/

[27] http://www.peterjbentley.com/heartchallenge/

[28] https://archive.physionet.org/physiobank/database/sufhsdb/

[29] https://www.kaggle.com/kinguistics/heartbeat-sounds 